\title{
Effects of tissue hydration on nanoscale structural morphology and mechanics of individual Type I collagen fibrils in the Brtl mouse model of Osteogenesis Imperfecta
}

\author{
Arika D. Kemp ${ }^{a}$, Chad C. Harding ${ }^{a}$, Wayne A. Cabral ${ }^{b}$, Joan C. Marini ${ }^{b}$, and Joseph M. \\ Wallace $^{\mathrm{a},{ }^{*}}$ \\ aDepartment of Biomedical Engineering, Indiana University-Purdue University at Indianapolis, \\ Indianapolis, IN, USA \\ bBone and Extracellular Matrix Branch, The Eunice Kennedy Shriver National Institute of Child \\ Health and Human Development (NICHD), NIH, Bethesda, MD, USA
}

\section{Abstract}

Type I collagen is the most abundant protein in mammals, and is a vital part of the extracellular matrix for numerous tissues. Despite collagen's importance, little is known about its nanoscale morphology in tissues and how morphology relates to mechanical function. This study probes nanoscale structure and mechanical properties of collagen as a function of disease in native hydrated tendons. Wild type tendon and tendon from the Brtl/+ mouse model of Osteogenesis Imperfecta were investigated. An atomic force microscope (AFM) was used to image and indent minimally-processed collagen fibrils in hydrated and dehydrated conditions. AFM was used because of the ability to keep biological tissues as close to their native in situ conditions as possible. The study demonstrated phenotypic difference in Brtl/+ fibril morphology and mechanics in hydrated tendon which became more compelling upon dehydration. Dried tendons had a significant downward shift in fibril D-periodic spacing versus a shift up in wet tendons. Nanoscale changes in morphology in dry samples were accompanied by significant increases in modulus and adhesion force and decreased indentation depth. A minimal mechanical phenotype existed in hydrated samples, possibly due to water masking structural defects within the diseased fibrils. This study demonstrates that collagen nanoscale morphology and mechanics are impacted in Brtl/+ tendons, and that the phenotype can be modulated by the presence or absence of water. Dehydration causes artifacts in biological samples which require water and this factor must be considered for studies at any length scale in collagen-based tissues, especially when characterizing disease-induced differences.

\section{Keywords}

AFM; Ultrastructure; Genotype/phenotype; D-periodic spacing; Modulus; Energy

\section{Introduction}

Type I collagen is the most abundant protein in mammals (Kadler et al., 1996). It is found throughout the body and forms a major portion of the extracellular matrix of many tissues including tendon, skin, arterial walls, bone and dentin. Type I collagen is a versatile protein

(C) 2012 Elsevier Inc. All rights reserved.

*Corresponding author. Address: Indiana University-Purdue University at Indianapolis, Department of Biomedical Engineering, 723 W Michigan St. SL220D, Indianapolis, IN 46202, USA. Fax: +1 317278 2455. jmwalla@iupui.edu (J.M. Wallace). 
that provides a scaffolding for formation of many tissues and a template for subsequent mineralization in bone and dentin. Most notably, it is the principal source of tensile strength in many tissues (Canty and Kadler, 2005).

Type I procollagen is a heterotrimer of three a chains (two a 1 and one a 2) wound into a right handed triple helix. The individual alpha chains are left-handed helical structures which have an uninterrupted motif of Glycine-X-Y triplets (X and Y are often proline and hydroxyproline, respectively) (Kadler et al., 2007). As an amino acid with a single hydrogen side chain, glycine's presence as every third residue is crucial, as it is the only amino acid which can be accommodated in the internal aspect of the collagen helix. Once the procollagen molecule is secreted from the cell, the non-helical propeptide ends are enzymatically cleaved releasing a mature collagen-molecule that is $300 \mathrm{~nm}$ long and $1.5 \mathrm{~nm}$ in diameter. Collagen self-assembles into a staggered, parallel twist to form a microfibril (Hulmes and Miller, 1979; Orgel et al., 2006; Smith, 1968; Wess et al., 1998), and microfibrils pack together to produce a three dimensional collagen fibril. This nanoscale fibril is the primary building block of many collagen-based tissues. Due to space between the ends of these molecules and an offset from row to row, gap and overlap zones exist within the fibril and produce an oscillating surface with a characteristic axial repeat pattern called the D-periodicity. Recent work has demonstrated that rather than being a singular value of $67 \mathrm{~nm}$ postulated by Hodge and Petruska (1963), the D-spacing exists as a distribution of values in collagen-based tissues (Fang et al., 2012; Wallace et al., 2010b). In bone, these distributions are altered with disease and may serve as an early disease marker (Wallace et al., 2011, 2010a).

Osteogenesis Imperfecta (OI) is a generalized disorder of connective tissues whose predominant symptoms and findings occur in bone, although multiple other tissues are impacted. Autosomal dominant OI types II, III and IV result primarily from point mutations causing the substitution of a triple-helical glycine $(80 \%)$ or splice site mutations $(20 \%)$ in the genes encoding the Type I collagen a chains (Marini et al., 2007). The disease displays a wide spectrum of phenotypes whose relationship to different mutations is incompletely understood although over 1500 independent alterations in the primary structure of collagen have been identified. Changes in a chain structure cause delayed protein folding, overmodification of the triple helix and decreased collagen quality (Glorieux et al., 2002). Although it is clear that these molecular changes are responsible for altered mechanical properties associated with OI (Byers et al., 1991; Fratzl et al., 1996; Jepsen et al., 1997; Kozloff et al., 2004), the link between nanoscale and tissue-level structure and function remains elusive.

Several studies have investigated the link between genotype and collagen structure in OI bone (Cassella et al., 1994; Sarathchandra et al., 1999; Wallace et al., 2011) and tendon (McBride et al., 1997; Misof et al., 1997), but little is known about collagen's nanoscale morphology in situ and how morphology relates to nanoscale mechanical function. The Brittle (Brtl/+) mouse, a model of Type IV OI, has a classical single glycine substitution (a cysteine is substituted for a glycine at the 349 position of the triple helix) in one Colla 1 allele. This model was created to study how a defined change in the amino acid sequence of collagen can lead to an OI phenotype (Forlino et al., 1999). A previous study in Brtl/+ bone at 2 months of age demonstrated a change in the distribution of D-spacing values (Wallace et al., 2011). This observation was important, but the mechanism leading to this change is unknown. The presence of mineral further complicates the interpretation of the results. In addition, the study was performed in air on dried bone samples. Collagen exists in a hydrated environment in vivo, and it is well known that collagen denatures (at least partially) when dehydrated (Saitô and Yokoi, 1992). This important issue of tissue hydration is particularly relevant when considering how dehydration impacts the mechanical 
properties of the collagen fibrils (Grant et al., 2008, 2009). Despite a wealth of knowledge concerning the effects of dehydration in collagen, many papers continue to investigate properties of collagen-based tissues in fixed, dehydrated or otherwise altered/manipulated samples and draw broad conclusions based on these studies.

The primary purpose of this study was to investigate the nanoscale structure and mechanical function of non-mineralized tendon in the Brtl/+ mouse model of OI. Under hydrated conditions, AFM was used to image and indent minimally-processed Type I collagen fibrils. The hypothesis was that like in bone, nanoscale morphology would be altered as a function of the disease. These changes in structure would be directly related to alterations in nanoscale mechanical integrity. Upon dehydration, it was further hypothesized that morphology, mechanics and their relationships with one another would change, altering the phenotype of the diseased tendons.

\section{Materials and methods}

\subsection{Animals and tissue preparation}

Brtl/+ and wild type (WT) mice from the mixed Sv129/CD-1/C57BL/6S background strain were used at 6 months of age with prior approval of the NICHD Animal Care Committee (protocol \# ASP 09-023). Four mice from each genotype were sacrificed by lethal injection, and tails were removed whole and processed for imaging. The skin of each tail was pulled back at the base and removed in a base-to-tip direction to expose the underlying tendon bundles. From each tail, $75 \mathrm{~mm}$ lengths of individual fascicles were removed and placed in phosphate buffered saline (PBS). Each fascicle was rinsed in water, placed on a glass slide and flattened with curved forceps. For fluid imaging, the sample was allowed to dry just enough to adhere to the glass, then was submerged in water and imaged. For imaging in air, samples were dried in ambient conditions and imaged.

\subsection{Atomic force microscopy (AFM) imaging and analysis}

Samples were imaged using a Bruker Catalyst AFM, operating in peak force tapping mode. Prior to imaging, each probe was calibrated. The probe was pushed into a glass surface and the deflection of the cantilever was measured to determine the cantilever's deflection sensitivity $(\mathrm{nm} / \mathrm{V})$. This value determines how much the cantilever will deflect under a given load. Next, the spring constant $(\mathrm{N} / \mathrm{m})$ of the cantilever was determined using the thermal tuning method. Finally, the radius of curvature was determined using a tip calibration sample. The fibrils became stiff and brittle when dried, necessitating the use of different imaging probes in wet and dry conditions (Grant et al., 2008). For wet imaging and indentation, ScanAsyst Fluid+ probes were used (Bruker, radius nominally $2 \mathrm{~nm}, k=0.7 \mathrm{~N} /$ $\mathrm{m}$ ). For dry imaging and indentation, stiffer RTESPA probes were used (Bruker, radius nominally $8 \mathrm{~nm}, k=40 \mathrm{~N} / \mathrm{m}$ ).

Making absolute $\mathrm{x}-\mathrm{y}$ distance measurements with AFM is limited by the calibration of the system. Calibration of the Bruker Catalyst AFM was performed using a $100 \times 100 \mathrm{~nm}$ calibration standard (Nanosensors 2D-100 standard). The scan size was set at $5 \mu \mathrm{m}(50 \times 50$ pitches) with $512 \times 512$ pixels and a scan rate of 0.5 lines/s. After calibration, the percentage error of the AFM was less than $1 \%$ in both the fast scan and slow scan directions.

For measurements of collagen morphology, $5 \times 5 \mu \mathrm{m}$ images were acquired from 3 locations in each of 2-3 fascicles per animal. At each location, peak force error images were analyzed to investigate the D-periodic spacing of individual fibrils using a two dimensional Fast Fourier Transform (2D FFT), as previously described (Wallace et al., 2011, 2010a,b). Briefly, analysis was performed on 10-15 individual fibrils per location, an average of $n=$ 65 per sample. A rectangular region of interest (ROI) was chosen along straight segments of 
individual fibrils (SPIP v5.1.5, Image Metrology; Hørsholm, Denmark). A 2D FFT was performed and the primary peak from the power spectrum was analyzed to determine the value of the D-periodic spacing for that fibril.

\subsection{AFM-based indentation}

Once an image was obtained, the AFM cantilever/probe was used as an indentation device. Four to five locations along the length of individual fibrils were indented to $20 \mathrm{nN}$ (wet) or $50 \mathrm{nN}$ (dry), and force-separation curves were acquired (Fig. 1). These indents were spaced apart by at least $10 \mathrm{D}$-periodic units to ensure that there was no interaction from one indent to the next. In addition, all indents were targeted toward the overlap region of the fibril for consistency, as others have shown variations in the mechanical properties of collagen fibrils between the gap and overlap regions (Balooch et al., 2008). On average, 25-30 individual fibrils were indented from each sample for an average of between 125 and 150 total indentations per sample.

Indentation depth $(\delta)$, energy dissipation and adhesion force were determined directly from each indentation curve. Several methods based on classical Hertz contact mechanics exist to calculate indentation modulus $\left(E_{\mathrm{S}}\right)$ from the unloading curve of AFM-based indentation data. Recent studies have utilized an extension of the Oliver Pharr method (Stolz et al., 2004, 2010), a technique which is well suited for instrumented nano-indentation studies where a blunt diamond tip is pushed into a surface (Oliver and Pharr, 1992). However, the majority or AFM-based indentation literature (and available software packages for analyzing AFM data) use either the classic Hertz model or the Sneddon model to extract elastic modulus data. In dry conditions, indentation depth was much smaller than the radius of curvature of the probe. Therefore, the classic Hertz model of contact between a rigid sphere and an elastic half space was used.

$$
F=\frac{4}{3} \cdot \frac{E_{s}}{1-v_{s}^{2}} \cdot \sqrt{r} \cdot \delta^{\frac{3}{2}}
$$

In Eq. (1), $F$ is the force and $r$ is the radius of curvature of the probe. Poisson's ratio $\left(\nu_{\mathrm{s}}\right)$ is not fully understood for collagen and as such, a value of 0.35 was chosen for all samples. When fibrils were wet, there was much more indentation depth despite the smaller applied forces. Therefore, the Hertz model is no longer valid and the Sneddon model of contact between a rigid cone and an elastic half space was used.

$$
F=\frac{2}{\pi} \cdot \frac{E_{s}}{1-v_{s}^{2}} \cdot \tan \alpha \cdot \delta^{2}
$$

In Eq. (2), $a$ is the opening angle of the AFM probe ( $a=17.5^{\circ}$ for the probes used here). When calculating modulus using either approach, irregular adhesion forces between the tip and sample dominate the lower-force and negative regions of the force displacement curve leading to decreased signal-to-noise and inaccuracies in the data fit. Preliminary studies indenting many wet and dry collagen samples were performed and analyzed to determine how much of the unloading curve to include in the analysis. Taking into account noisiness in the data, the variability in adhesion forces and the reproducibility of the data fit, data from the middle $50 \%$ of the unloading curve were included.

\subsection{Statistical analysis}

All statistical analyses utilized SPSS (Version 19.0, IBM). For all investigations, a value of $p<0.05$ was considered significant. To investigate the effect of genotype on morphology, 
D-periodic spacing values measured from an individual tail sample were averaged, yielding a single value for that sample. The values from WT and Brtl/+ mice were then compared using a Student's $t$-test in wet or dry conditions ( $n=4$ per condition). To investigate differences in distributions of fibril morphology between genotypes in wet or dry conditions, the histogram and the Cumulative Distribution Function (CDF) of each group were computed. The CDF shows the percentage of a given sample population which is contained up to a particular value, easily demonstrating differences between distributions in both mean and standard deviation. To test for statistically significant differences between distributions, Kolmogorov-Smirnov (K-S) tests were applied to the data ( $n=255$ for WT wet, $n=244$ for Brtl/+ wet, $n=257$ for WT Dry, $n=291$ for Brtl/+ Dry).

Mechanical comparisons were made in one of three ways. First, as was the case for morphology above, values measured from an individual tail sample were averaged, yielding a single value for that sample for each mechanical parameter. The values from WT and Brtl/ + mice were then compared using a Student's t-test in wet and dry conditions $(n=4$ per condition). Next, each property from all indents within each genotype/condition were displayed as histograms and CDFs ( $n=555$ for WT wet, $n=614$ for Brtl/+ Wet, $n=464$ for WT dry, $n=479$ for Brtl/ + dry). To test for statistically significant differences between distributions, Kolmogorov-Smirnov (K-S) tests were applied to each property. Final mechanical investigations were performed by averaging the 4-5 indents made in a given fibril to yield a single value for that fibril ( $n=112$ for WT wet, $n=124$ for Brtl/+ Wet, $n=$ 101 for WT dry, $n=109$ for Brtl/+ dry). In an attempt to relate morphology to mechanics, linear correlation analyses were performed between D-spacing and mechanical properties.

\section{Results}

\subsection{Morphological changes in hydrated Brtl/+ tail tendon fibrils}

The D-periodic spacing of individual Type I collagen fibrils was used as the metric of fibril nanoscale morphology. To assess the D-periodic spacing in murine tail tendon as a function of the $\mathrm{Brtl} / \mathrm{+}$ OI genotype, tails from 6 month old male mice were used. Images were acquired from 3 locations in each of 2-3 fascicles per animal (Fig. 2). 2D Fast Fourier Transforms were then performed on 10-15 individual fibrils. All measurements within each tendon samples were averaged to yield the mean fibril spacing for that tail, then the $4 \mathrm{WT}$ values were compared with the 4 Brtl/+ values (Fig. 3). For the hydrated samples, the overall mean values were $68.2 \pm 1.2$ and $67.5 \pm 1.4 \mathrm{~nm}$ for $\mathrm{WT}$ and Brtl//+, respectively. There was no significant effect of genotype on mean fibril spacing in the wet samples ( $p=$ $0.123)$.

Important information can also be obtained by analyzing the population distribution of fibril morphologies within each genotype (Fig. 4). Fig. 4a shows that a distribution of D-period spacing exists in each genotype and that the Brtl/+ distribution is shifted to lower spacing values versus the WT distribution. The main population of fibrils in each group was defined using the mean of the WT group \pm 1 standard deviation. Within this range, $72 \%$ of WT fibrils and $67 \%$ of Brtl/+ fibrils are found. Below this range, $14.5 \%$ of WT fibrils were found in comparison to $29 \%$ of Brtl/+ fibrils, indicating that the population distribution of Brtl/+ fibrils was shifted downwards versus WT. As a second method to visualize these distributions, the Cumulative Distribution Function (CDF) was computed from the measurements in each group (Fig. 4b). Both the histogram and the CDF indicate that the $\mathrm{Brtl} /++$ tendon populations were shifted downward in comparison to their WT counterparts, although the shape of the two populations were similar. A Kolmogorov-Smirnov (K-S) test, a test which is sensitive to changes in both the mean value and standard deviation of a distribution, indicated that the D-periodic spacings in Brtl/+ tendons were significantly different than the WT tendons $(p<0.001)$. 


\subsection{Morphological changes in dehydrated Brtl/+ tail tendon fibrils}

For the dehydrated samples, the overall mean values were $68.6 \pm 0.8$ and $68.9 \pm 1.0 \mathrm{~nm}$ for WT and Brtl/+, respectively (Fig. 3). There was no significant effect of genotype on mean fibril spacing in the dry samples $(p=0.209)$. In Fig. 5, a clear difference between population distributions is not immediately apparent, although the Brtl/+ population may be slightly shifted upwards. The main population of fibrils, again defined as the mean of the WT group \pm 1 standard deviation, contained $70 \%$ of WT fibrils but only $62.5 \%$ of Brtl/+ fibrils. In this case, $17.5 \%$ of WT were above this range compared to $26.5 \%$ of Brtl/+ fibrils. The CDF indicates that the populations are similar until about $68 \mathrm{~nm}$, then the WT population increases rapidly while the Brtl/+ population is delayed, spreading Brtl/+ values towards larger spacing. A K-S test indicated that the D-periodic spacings were significantly different between the two groups $(p<0.001)$.

\subsection{Mechanical changes in hydrated Brtl/+ tail tendon fibrils}

Mechanical changes in Type I collagen fibrils were assessed using the AFM as an indentation device. Four to five locations along the length of individual fibrils were indented to a maximum force of $20 \mathrm{nN}$. These indents were spaced apart by at least $10 \mathrm{D}$-periodic units to ensure that there was no interaction from one indent to the next. In addition, all indents were targeted toward the overlap region of the fibril for consistency, as others have shown variations in the mechanical properties of collagen fibrils between the gap and overlap regions (Balooch et al., 2008). On average, 30 individual fibrils were indented from each animal for an average of approximately 150 total indentations per sample.

Mechanical properties including elastic modulus, indentation depth, energy dissipation and adhesion force were analyzed. For the first analysis, all measurements within each tendon sample were averaged to yield the mean value from each property for that tail, then the 4 WT values were compared with the $4 \mathrm{Brtl} /+$ values (Table 1 ). Surprisingly, there were no significant differences in any of the analyzed properties between the WT and Brtl/+ groups.

The next mechanical comparisons were made for all indentations within each genotype, leading to a samples size of $n=555$ for WT and $n=614$ for Brtl/t. Histograms and CDFs were also produced for each property and statistically compared using KS tests (Fig. 6). As was the case at the sample level, there were no significant differences in mean between WT and $\mathrm{Brtl} /++$ elastic modulus, indentation depth or energy dissipation despite the large samples sizes (data not shown). There were similarly no differences in distributions for these properties (Fig. 6). There was, however, a significant difference in adhesion force (the maximum attractive (negative) force between probe and sample upon withdrawal of the probe, Fig. 1). In the Brtl/+ samples, the values climb more quickly with $90 \%$ of the Brtl/+ values being less than or equal to $0.5 \mathrm{nN}$ versus the WT values which do not reach $90 \%$ until $1.5 \mathrm{nN}$. The WT population also ends at $2.7 \mathrm{nN}$, while a tail exists in the Brtl/+ population out to more than $6 \mathrm{nN}$.

Final mechanical investigations were performed by pooling the 4 to 5 indents made in a given fibril to yield a single value for that fibril, resulting in a sample size of $n=112$ for WT and $n=124$ for Brtl. In an attempt to relate morphology to mechanics, linear correlation analyses were performed between D-spacing and mechanical properties (Table 2). Weak and non-significant relationships were found for most properties, with the exception of a significant negative relationship between energy dissipation and D-spacing in the WT samples. Energy dissipation was also significantly correlated with modulus (negative) and deformation (positive) in both WT and Brtl/+ fibrils. Finally, a strong and positive relationship existed between energy dissipation and adhesion force, suggesting that adhesion may be the mechanism behind dissipated energy with loading (Fig. 7A). 


\subsection{Mechanical changes in dehydrated Brtl/+ tail tendon fibrils}

As was the case for wet conditions, four to five locations along the length of individual fibrils were indented in the dry fibrils. Because of a stiffening of these fibrils with dehydration, a higher force of $50 \mathrm{nN}$ was needed to get enough deformation of the fibrils for analysis. All measurements within each tendon sample were averaged to yield the mean value from each property for that tail, then the $4 \mathrm{WT}$ values were compared with the $4 \mathrm{Brtl} /+$ values (Table 3). As was the case in the wet samples, there were no significant differences in mean values for any of the mechanical properties between WT and Brtl/+ samples. There was a marginal decrease in indentation depth and a similar increasing trend in modulus in the $\mathrm{Brtl} /+$ samples.

The next mechanical comparisons were made for all indentations within each genotype, leading to a samples size of $n=464$ for WT and $n=479$ for Brtl/+. Histograms and CDFs were produced for each property (Fig. 8). As opposed to the wet condition, there were significant mean differences between the WT and Brtl/+ fibrils for elastic modulus, indentation depth and adhesion force, but no difference for energy dissipation $(p<0.001$ for all, data not shown). There were similar significant differences in population distributions (Fig. 8). Population differences were particularly compelling for modulus and indentation depth, where the Brtl/+ fibrils were, on average, $37 \%$ stiffer leading to $23 \%$ lower indentation depth for the same indentation force.

Pooling the 4-5 indents made in a given fibril to yield a single value for that fibril resulted in a sample size of $n=101$ for WT and $n=109$ for Brtl/t. Linear correlations were performed between D-spacing and mechanical properties (Table 4). In the WT samples, there was no relationship between D spacing and any mechanical measure. The Brtl/+ samples showed strong significant correlations between D-spacing and both modulus and indentation depth, with a marginal negative relationship between D-spacing and energy dissipation. There were also strong relationships between energy dissipation and both modulus and indentation depth in the Brtl/+ fibrils. As was the case in dry fibrils, a strong and positive relationship existed between energy dissipation and adhesion force, again indicating that adhesion is the primary mechanism behind lost energy with loading (Fig. 7B).

As a means to understand how the D-periodic spacing and mechanical properties may be related to one another in general, linear correlation analyses were performed on data from all individual fibrils. In total, 112 WT wet, $124 \mathrm{Brtl} /+$ wet, $101 \mathrm{WT}$ Dry and $109 \mathrm{Brtl} /+$ fibrils were analyzed, making the total sample size of $n=446$ fibrils (Table 5). All measured mechanical properties had strong significant relationships with D-spacing with the exception of energy dissipation. There were also strong significant relationships between energy dissipation and all other mechanical properties. One relationship of note was between adhesion force and energy dissipation. The correlation coefficient for this comparison was 0.832 , resulting in an $r^{2}=0.692$, suggesting that nearly $70 \%$ of the variation in energy dissipation can be explained by adhesion force.

\section{Discussion}

This study's main goal was to investigate the nanoscale structure and mechanical function of normal tendon fibrils and fibrils from the Brtl/+ mouse model of Osteogenesis Imperfecta under hydrated conditions. However, since many papers in a variety of journals dealing with collagen-based tissues continue to investigate properties in fixed, dehydrated or otherwise altered/manipulated samples, a secondary goal of this study was to determine how the Brtl/+ phenotype changes upon drying of the tissues. AFM was used to image and indent minimally-processed Type I collagen fibrils in wet and dry conditions. As noted in Brtl/+ 
bone (Wallace et al., 2011), phenotypic changes in the fibril ultrastructure were detected in tendon. In wet samples, the population distribution of D-periodic spacing was shifted downward, but was not accompanied by nanoscale mechanical changes. The phenotype differed in dried samples where the population of D-period spacings was shifted upward in $\mathrm{Brtl} /++$ tendons versus controls, and was accompanied by significant increases in modulus and adhesion force and decreased indentation depth. Further investigations into the mechanism behind these alterations in morphology and mechanics are currently under way.

Several groups have used AFM to study hydrated collagen (Bozec and Horton, 2005; Cisneros et al., 2006; Grant et al., 2009; Yang et al., 2008), but few have performed systematic work in situ in native tissues (Balooch et al., 2008; Baselt et al., 1993; Raspanti et al., 2001) (i.e. collagen that was not acid solubilized and reconstituted). Collagen absorbs water and swells, so imaging in fluid often leads to degraded image resolution and quality, making it difficult to quantitatively assess images. In addition, the D-period spacing is often not resolvable when fibrils are wet (Baselt et al., 1993). There were qualitative differences in wet versus dry tendons in this study (Fig. 2). Fibrils were flatter and wider when dry for both WT (A vs. B) and Brtl/+ (C vs. D) samples. Fibrils were almost always packed together in the lateral direction with no space between adjacent fibrils, making it impossible to accurately measure the width of individual fibrils since the entire width was not exposed. It was also not possible to measure fibril height since there was no exposed substrate to define as zero, and no way of knowing if the fibrils were in a single layer. Regardless, flatter and wider fibrils in dry versus wet samples were consistently observed. In addition, a variety of objects were present on the surface of wet fibrils (Fig. 2A and C). These objects are assumed to be other non-collagenous proteins and fragments of the endotenon which surrounds each fascicle (Jozsa et al., 1991). Because of their movement, these loose objects presented another challenge to the imaging of hydrated fibrils.

The D-period has been used to characterize collagen in a variety of tissues (Wallace et al., 2010b), and to demonstrate tissue changes with disease (Fang et al., 2012; Wallace et al., 2011, 2010a). Here, the D-period distribution of Brtl/+ fibrils was shifted downward versus WT when imaged wet, but distribution shapes were similar (Fig. 4). This downward shift was driven by a greater number of fibrils with shorter spacings. In fact, $30 \%$ of Brtl/+fibrils had spacings less the mean minus 1 standard deviation of the WT population. When dried, the D-spacing phenotype was different with the Brtl/+ population shifted up relative to WT (Fig. 5). This change in dried fibrils is not the same as previously reported in Brtl/+bone (Wallace et al., 2011). The discrepancy could be due to the presence or absence of mineral or the difference in animal age between the studies. Both possibilities will be explored in future experiments.

The reason for hydration dependence in the morphological phenotype may be found in the fact that there was a differential change in WT versus Brtl/+ fibrils as they dried (Supplemental Fig. S1a). In WT, there was a non-significant $0.4 \mathrm{~nm}$ increase in D-spacing with drying ( $n=4$ per group; WT Wet: $68.2 \pm 1.2 \mathrm{~nm}$; WT Dry: $68.6 \pm 0.8 \mathrm{~nm}, p=0.200$ ). This change in WT fibrils was driven by a loss of fibrils with spacing less than $66 \mathrm{~nm}$ which caused the WT population to become more uniform. In comparison, the entire Brtl/+ population shifted up to higher spacing values when dried (Supplemental Fig. S1b). The mean change was a significant $1.4 \mathrm{~nm}$ increase in dry Brtl/+ fibrils ( $n=4$ per group, Brtl/+ Wet: $67.5 \pm 1.4 \mathrm{~nm}$; Brtl/+ Dry: $68.9 \pm 1.0 \mathrm{~nm} p<0.001$ ). This differential change with drying could be indicative of an alteration of the internal structure of Brtl/+ fibrils. Water may facilitate bridging around and within collagen fibrils which tightens/compresses the structure leading to a population with more shorter-spaced fibrils (Bella et al., 1995). This condition can be thought of causing a pre-stress within the fibrillar structure. Altered internal structure in Brtl/+ fibrils may be the reason why the Brtl/+ population is shifted 
down versus the WT populations when wet, as these fibrils may be more easily compressed by the presence of water (Fig. 4). As water leaves and bridging is lost, the compressed fibrils relax and expand. Fibrils that are initially more compressed may have more of an ability to expand versus fibrils already in an expanded confirmation causing a loss of fibrils with shorter spacing in both the WT and Brtl/+ samples. Since the Brtl/+ population had more fibrils with shorter spacings versus WT in the wet state (Fig. 4), this could account for the differential change with drying. A second possible impact may explain the observation here and elsewhere that fibril height is decreased with dehydration (Grant et al., 2009). The Poisson Effect states that shrinkage in the $z$-direction (height) should be accompanied by expansion in the radial $(y)$ and axial $(x)$ directions (Beer et al., 2008). The combination of decreased height ( $z$ direction), increased width ( $y$ direction) and increased D-spacing ( $x$ direction) with dehydration are all consistent with this effect.

A goal of the current study was to understand how the collagen D-spacing changes as a function of hydration. Previous work in dentin showed that drying causes a relatively narrow distribution of D-spacing values to shift to lower values and to split into sub-populations (Habelitz et al., 2002). Results here differed from this previous work. In WT and Brtl/+ fibrils, populations became more uniform and shifted towards higher spacing values with drying (Supplemental Fig. S1). The reason for these conflicting results may be the way in which D-spacing was measured (2D FFT here versus 1D FFT in the previous work). Another possibility is that mineral in dentin could be an important factor in determining Dspacing. A final possibility is that fibrils here were imaged in water while the previous study imaged in a balanced salt solution. Mechanical properties of individual fibrils are influenced by salt concentration (Grant et al., 2009), so the lack of salt in the current study may alter the D-spacing as well.

Mechanical differences between WT and Brtl/+ fibrils also had divergent phenotypes in dry and wet conditions. Under hydrated conditions, the only convincing difference between Brtl/ + and WT samples was in the population distribution of adhesion force (Fig. 6D). The Brtl/+ population was shifted to lower adhesion forces than the WT population with a long tail towards higher forces. Adhesion was a driving force behind energy dissipation with loading (Fig. 7), an important observation which could be indicative of changes at the surface of the diseased fibrils. However, the effect was mild and not related to other parameters. When dried, the phenotype was more compelling for all mechanical properties (Fig. 8). A population shift towards higher modulus in Brtl/+ fibrils led to decreased indentation depth versus WT fibrils ( $p<0.001$ for both). However, a comparison of the mean values averaged over each sample ( $n=4$ per genotype) showed a non-significant difference for both properties ( $p=0.129$ for modulus, $p=0.083$ for indentation depth). Given the $37 \%$ increase in modulus in the Brtl/+ fibrils, the lack of significant difference is likely due to variance in the data. A post hoc power analysis indicated that for a value of $a=0.05$ and a power of 0.80 , a sample size of $n=13$ mice would be required. This sample size is not realistic for an AFM study, but future studies will use this information to increase the probability of detecting mean differences in mechanical properties.

It is postulated that the lack of mechanical phenotype in wet fibrils versus the compelling and significant phenotype in dry fibrils is related to detrimental alterations to the internal structure of the Brtl/+ fibrils discussed above. In fluid, fibrils absorb water, become taller and pre-stressed. Water bridges the damaged structure and may be able to carry some of the compressive load as a fibril is indented, masking changes in structure which may be present. As water is lost, the damaged structure collapses driving a large change in D-spacing and the resulting mechanical changes compared with WT fibrils. Recent computational work indicated that free energy changes as a function of OI point mutations in model collagen molecules (Lee et al., 2011). Others have studied how amino acid sequence changes lead to 
packing differences in collagen molecules which may contribute to compromised mechanical integrity (Bella et al., 1994; Gautieri et al., 2009a,b). Although the model mechanical tests were performed in tension, their results are supportive of conclusions drawn here.

Some weaknesses in this study deserve noting. Indentations were in the z-direction but Dspacing is an axial measure, a difference which may explain why relationships between Dspacing and mechanical properties were weak (Tables 2 and 4). Future studies will investigate other methods to test individual fibrils in the axial direction using AFM-based approaches (Graham et al., 2004; Svensson et al., 2010; Yang et al., 2008). Because of fibril stiffening with drying, different probes, loads and contact mechanics models were needed for wet and dry samples (Grant et al., 2008). Despite these limitations, significant changes in mechanical properties did occur with drying in each genotype. As an example, there was a two order of magnitude increase in modulus with drying in both genotypes (Supplemental Fig. S2). These limitations may make it challenging to directly compare mechanical properties from wet and dry fibrils using AFM alone, but the techniques did uncover important changes in nanoscale morphology and mechanical properties with disease.

In conclusion, this study in minimally processed tendons demonstrated significant phenotypic changes in Brtl/+ collagen fibril morphology and mechanics, a phenotype which is altered upon dehydration of the tissue. Morphological phenotypes differed in wet and dry samples, with dried tendons displaying a more compelling downward shift in fibril Dperiodic spacing versus a shift up in wet tendons. A nanoscale change in morphology in dry samples was accompanied by significant increases in modulus and adhesion force and decreased indentation depth. Little mechanical phenotype existed in hydrated samples, possibly due to water masking structural defects within the diseased fibrils. This study demonstrates that morphological and mechanical changes that are present in this collagenbased disease model are altered upon dehydration of the tissue. Dehydration and other manipulations to native tissues can cause artifacts in biological samples which require water. This factor must be considered for studies at any length scale in collagen-based tissues, especially when characterizing disease-induced differences.

\section{Supplementary Material}

Refer to Web version on PubMed Central for supplementary material.

\section{Acknowledgments}

This work was partially supported by IUPUI Departmental Funds (JMW). JCM is supported by NICHD Intramural Funding.

\section{References}

Balooch M, Habelitz S, Kinney JH, Marshall SJ, Marshall GW. Mechanical properties of mineralized collagen fibrils as influenced by demineralization. Journal of Structural Biology. 2008; 162:404410. [PubMed: 18467127]

Baselt DR, Revel JP, Baldeschwieler JD. Subfibrillar structure of type I collagen observed by atomic force microscopy. Biophysical Journal. 1993; 65:2644-2655. [PubMed: 8312498]

Beer, FP.; Johnston, ER., Jr; DeWolf, JT.; Mazurek, DF. Mechnanics of Materials. 5. McGraw Hill; Boston: 2008.

Bella J, Brodsky B, Berman HM. Hydration structure of a collagen peptide. Structure. 1995; 3:893906. [PubMed: 8535783]

Bella J, Eaton M, Brodsky B, Berman HM. Crystal molecular structure of a collagen-like peptide at 1.9 A resolution. Science. 1994; 266:75. [PubMed: 7695699] 
Bozec L, Horton M. Topography and mechanical properties of single molecules of type I collagen using atomic force microscopy. Biophysical Journal. 2005; 88:4223-4231. [PubMed: 15778444]

Byers PH, Wallis GA, Willing MC. Osteogenesis imperfecta: translation of mutation to phenotype. Journal of Medical Genetics. 1991; 28:433-442. [PubMed: 1895312]

Canty EG, Kadler KE. Procollagen trafficking, processing and fibrillogenesis. Journal of Cell Science. 2005; 118:1341-1353. [PubMed: 15788652]

Cassella JP, Barber P, Catterall AC, Ali SY. A morphometric analysis of osteoid collagen fibril diameter in osteogenesis imperfecta. Bone. 1994; 15:329-334. [PubMed: 8068454]

Cisneros DA, Hung C, Franz CM, Muller DJ. Observing growth steps of collagen self-assembly by time-lapse high-resolution atomic force microscopy. Journal of Structural Biology. 2006; 154:232-245. [PubMed: 16600632]

Fang M, Liroff KG, Turner AS, Les CM, Orr BG, Holl MMB. Estrogen depletion results in nanoscale morphology changes in dermal collagen. Journal of Investigative Dermatology. 2012; 132:17911797. [PubMed: 22437310]

Forlino A, Porter FD, Lee EJ, Westphal H, Marini JC. Use of the Cre/lox recombination system to develop a non-lethal knock-in murine model for osteogenesis imperfecta with an alpha1(I) G349C substitution. Variability in phenotype in BrtlIV mice. The Journal of Biological Chemistry. 1999; 274:37923-37931. [PubMed: 10608859]

Fratzl P, Paris O, Klaushofer K, Landis WJ. Bone mineralization in an osteogenesis imperfecta mouse model studied by small-angle X-ray scattering. Journal of Clinical Investigation. 1996; 97:396402. [PubMed: 8567960]

Gautieri A, Vesentini S, Redaelli A, Buehler MJ. Single molecule effects of osteogenesis imperfecta mutations in tropocollagen protein domains. Protein Science. 2009a; 18:161-168. [PubMed: 19177360]

Gautieri A, Uzel S, Vesentini S, Redaelli A, Buehler MJ. Molecular and mesoscale mechanisms of osteogenesis imperfecta disease in collagen fibrils. Biophysical Journal. 2009b; 97:857-865. [PubMed: 19651044]

Glorieux FH, Ward LM, Rauch F, Lalic L, Roughley PJ, Travers R. Osteogenesis imperfecta type VI: a form of brittle bone disease with a mineralization defect. Journal of Bone and Mineral Research. 2002; 17:30-38. [PubMed: 11771667]

Graham JS, Vomund AN, Phillips CL, Grandbois M. Structural changes in human type I collagen fibrils investigated by force spectroscopy. Experimental Cell Research. 2004; 299:335-342. [PubMed: 15350533]

Grant CA, Brockwell DJ, Radford SE, Thomson NH. Effects of hydration on the mechanical response of individual collagen fibrils. Applied Physics Letters. 2008; 92:1-3.

Grant CA, Brockwell DJ, Radford SE, Thomson NH. Tuning the elastic modulus of hydrated collagen fibrils. Biophysical Journal. 2009; 97:2985-2992. [PubMed: 19948128]

Habelitz S, Balooch M, Marshall SJ, Balooch G, Marshall GW Jr. In situ atomic force microscopy of partially demineralized human dentin collagen fibrils. Journal of Structural Biology. 2002; 138:227-236. [PubMed: 12217661]

Hodge, AJ.; Petruska, JA. Recent studies with the electron microscope on ordered aggregates of the tropocollagen molecule. In: Ramachandran, GN., editor. Aspects of Protein Structure. Academic Press; New York: 1963. p. 289-306.

Hulmes DJS, Miller A. Quasi-hexagonal molecular packing in collagen fibrils. Nature. 1979; 282:878880. [PubMed: 514368]

Jepsen KJ, Schaffler MB, Kuhn JL, Goulet RW, Bonadio J, Goldstein SA. Type I collagen mutation alters the strength and fatigue behavior of Mov13 cortical tissue. Journal of Biomechanics. 1997; 30:1141-1147. [PubMed: 9456382]

Jozsa L, Kannus P, Balint J, Reffy A. Three-dimensional infrastructure of human tendons. Cells Tissues Organs. 1991; 142:306-312.

Kadler KE, Holmes DF, Trotter JA, Chapman JA. Collagen fibril formation. Biochemical Journal. 1996; 316:1-11. [PubMed: 8645190]

Kadler KE, Baldock C, Bella J, Boot-Handford RP. Collagens at a glance. Journal of Cell Science. 2007; 120:1955-1958. [PubMed: 17550969] 
Kozloff KM, Carden A, Bergwitz C, Forlino A, Uveges TE, Morris MD, Marini JC, Goldstein SA. Brittle IV mouse model for osteogenesis imperfecta IV demonstrates postpubertal adaptations to improve whole bone strength. Journal of Bone and Mineral Research. 2004; 19:614-622. [PubMed: 15005849]

Lee KH, Kuczera K, Banaszak Holl MM. The severity of osteogenesis imperfecta: a comparison to the relative free energy differences of collagen model peptides. Biopolymers. 2011; 95:182-193. [PubMed: 20945334]

Marini JC, Forlino A, Cabral WA, Barnes AM, San Antonio JD, Milgrom S, Hyland JC, Korkko J, Prockop DJ, De Paepe A. Consortium for osteogenesis imperfecta mutations in the helical domain of type I collagen: regions rich in lethal mutations align with collagen binding sites for integrins and proteoglycans. Human Mutation. 2007; 28:209-221. [PubMed: 17078022]

McBride DJ Jr, Choe V, Shapiro JR, Brodsky B. Altered collagen structure in mouse tail tendon lacking the alpha 2(I) chain. Journal of Molecular Biology. 1997; 270:275-284. [PubMed: 9236128]

Misof K, Landis WJ, Klaushofer K, Fratzl P. Collagen from the osteogenesis imperfecta mouse model (oim) shows reduced resistance against tensile stress. Journal of Clinical Investigation. 1997; 100:40-45. [PubMed: 9202055]

Oliver WC, Pharr GM. Improved technique for determining hardness and elastic modulus using load and displacement sensing indentation experiments. Journal of Materials Research. 1992; 7:15641583.

Orgel JPRO, Irving TC, Miller A, Wess TJ. Microfibrillar structure of type I collagen in situ. Proceedings of the National Academy of Sciences. 2006; 103:9001-9005.

Raspanti M, Congiu T, Guizzardi S. Tapping-mode atomic force microscopy in fluid of hydrated extracellular matrix. Matrix Biology. 2001; 20:601-604. [PubMed: 11731276]

Saitô H, Yokoi M. A 13C NMR study on collagens in the solid state: hydration/dehydration-induced conformational change of collagen and detection of internal motions. Journal of Biochemistry. 1992; 111:376-382. [PubMed: 1587801]

Sarathchandra P, Pope FM, Ali SY. Morphometric analysis of type I collagen fibrils in the osteoid of osteogenesis imperfecta. Calcified Tissue International. 1999; 65:390-395. [PubMed: 10541766]

Smith JW. Molecular pattern in native collagen. Nature. 1968; 219:157-158. [PubMed: 4173353]

Stolz M, Raiteri R, Daniels AU, VanLandingham MR, Baschong W, Aebi U. Dynamic elastic modulus of porcine articular cartilage determined at two different levels of tissue organization by indentation-type atomic force microscopy. Biophysical Journal. 2004; 86:3269-3283. [PubMed: 15111440]

Stolz M, Gottardi R, Raiteri R, Miot S, Martin I, Imer R, Staufer U, Raducanu A, Düggelin M, Baschong W. Early detection of aging cartilage and osteoarthritis in mice and patient samples using atomic force microscopy. Nature Nanotechnology. 2010; 5:186-192.

Svensson RB, Hassenkam T, Grant CA, Magnusson SP. Tensile properties of human collagen fibrils and fascicles are insensitive to environmental salts. Biophysical Journal. 2010; 99:4020-4027. [PubMed: 21156145]

Wallace JM, Orr BG, Marini JC, Banaszak Holl MM. Nanoscale morphology of Type I collagen is altered in the brtl mouse model of osteogenesis imperfecta. Journal of Structural Biology. 2011:146-152. [PubMed: 20696252]

Wallace JM, Erickson B, Les CM, Orr BG, Holl MMB. Distribution of type I collagen-morphologies in bone: Relation to estrogen depletion. Bone. 2010a; 46:1349-1354. [PubMed: 19932773]

Wallace JM, Chen QS, Fang M, Erickson B, Orr BG, Holl MMB. Type I collagen exists as a distribution of nanoscale morphologies in teeth, bones, and tendons. Langmuir. 2010b; 26:73497354. [PubMed: 20121266]

Wess T, Hammersley A, Wess L, Miller A. Molecular packing of type I collagen in tendon1. Journal of Molecular Biology. 1998; 275:255-267. [PubMed: 9466908]

Yang L, van der Werf KO, Fitié CFC, Bennink ML, Dijkstra PJ, Feijen J. Mechanical properties of native and cross-linked type I collagen fibrils. Biophysical Journal. 2008; 94:2204-2211. [PubMed: 18032556] 


\section{Appendix A. Supplementary data}

Supplementary data associated with this article can be found, in the online version, at http:// dx.doi.org/10.1016/j.jsb.2012.09.012. 


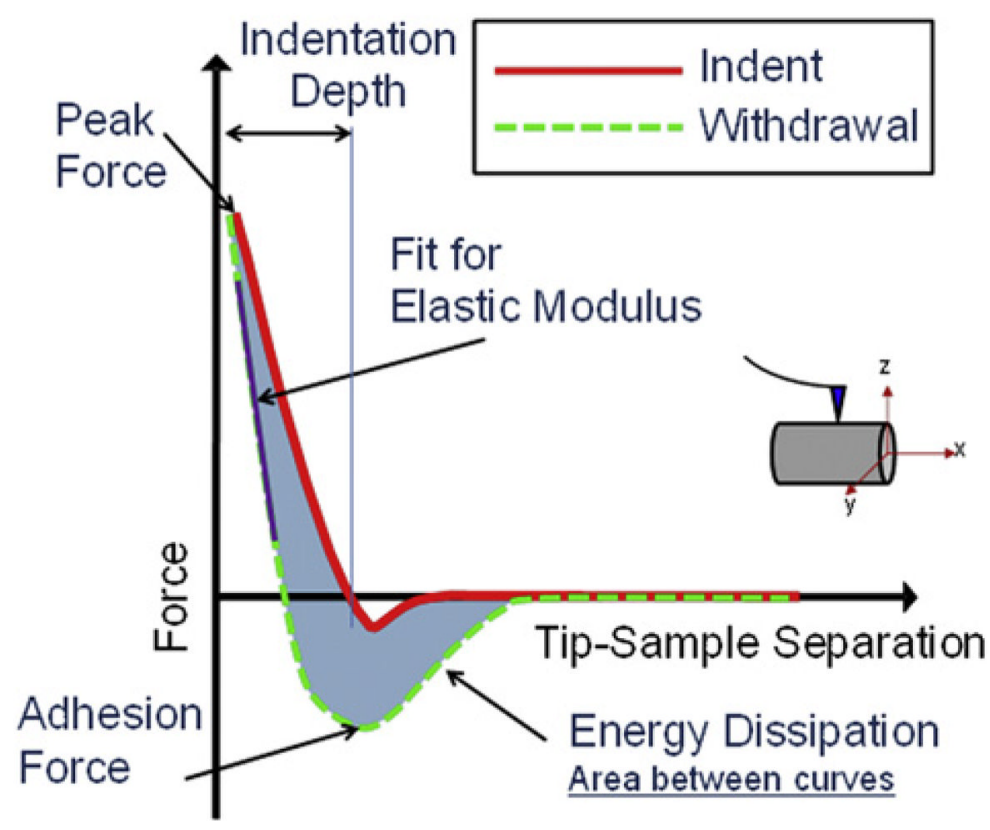

Fig. 1.

schematic force-separation curve. The AFM cantilever/probe was used as an indentation device. Four to five locations along the length of individual fibrils were indented to $20 \mathrm{nN}$ (wet) or $50 \mathrm{nN}$ (dry). From the resulting curve, indentation depth, energy dissipation and adhesion force were determined. Indentation modulus of the sample was calculated following a contact mechanics-based approach described in the text. 

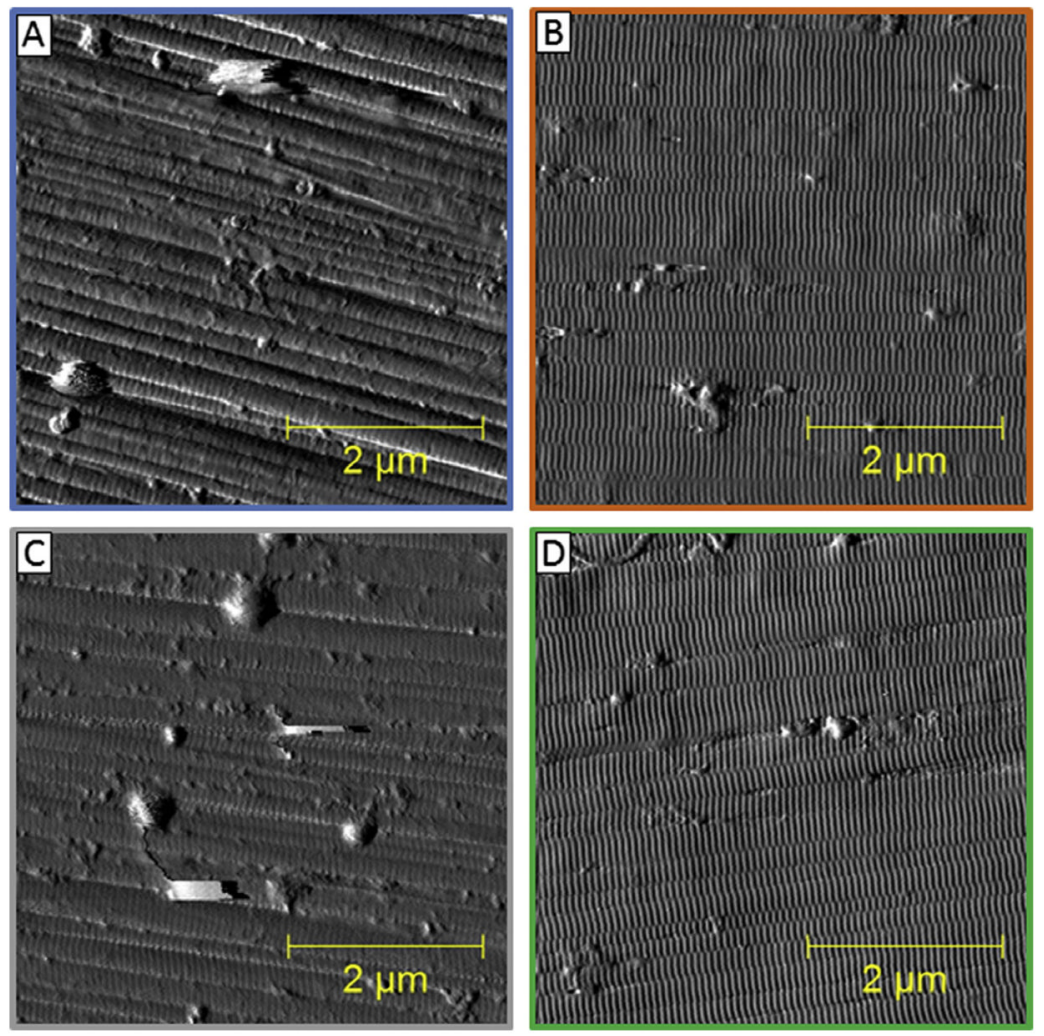

Fig. 2.

Representative images from hydrated and dehydrate tendon samples. Four groups were imaged in the current study, and are represented here in $5 \times 5 \mu \mathrm{m}$ atomic force microscope (AFM) images. Panel A is an image from the wild type (WT) wet group, Panel B is WT dry, panel C is Brtl/+ wet and panel D is Brtl/+ dry. Qualitatively, there were readily observable differences in wet versus dry tendon samples. Fibrils are flatter and wider in dry versus wet conditions for both the WT and Brtl/+ samples. A variety of other objects are apparent on the surface of fibrils when wet (A and C). These objects are assumed to be other noncollagenous proteins as well as fragments of the endotenon which surrounds each individual fascicle. 


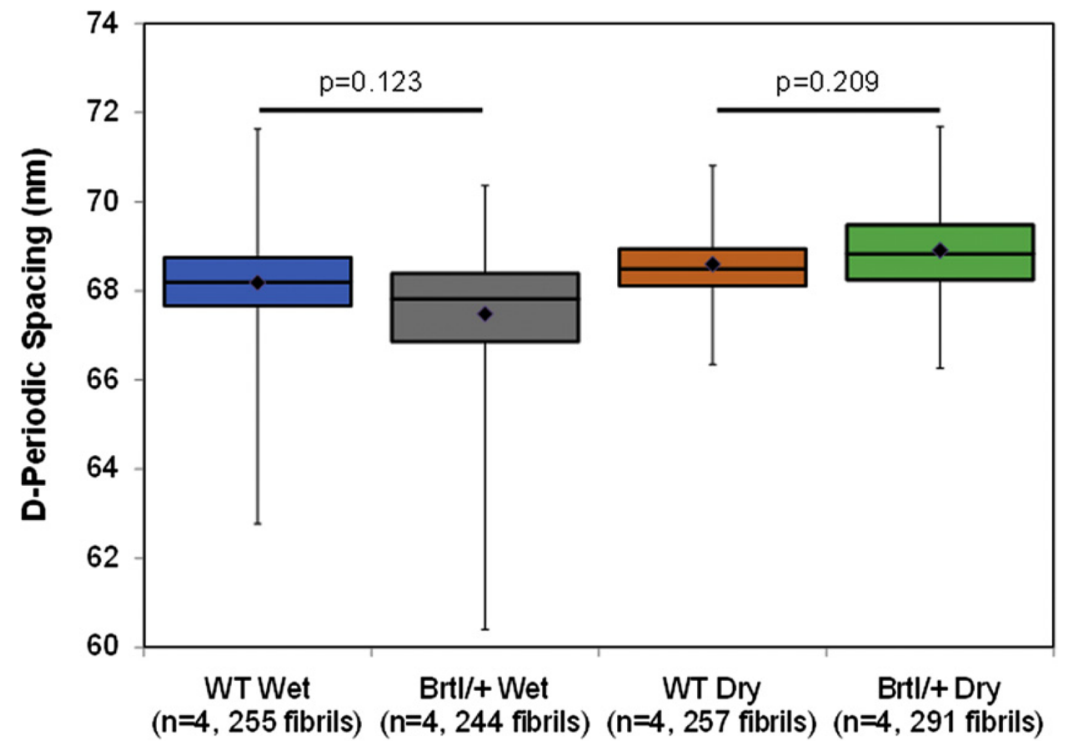

Fig. 3.

Boxplot representation of D-Periodic Spacing. The box is the interquartile region (middle $50 \%$ of the data), the horizontal line inside of the box is the median, and the diamond is the mean. The whiskers on the box are the minimum and maximum observation for that sample. The boxplot represents all measured fibrils from a given group. All measurements within each tendon samples were averaged to yield the mean fibril spacing for that tail, then the 4 wild type (WT) values were compared with the $4 \mathrm{Brtl} /+$ values in wet and dry conditions. The indicated $\mathrm{p}$ value is for these comparisons and indicates no significant difference between WT and Brtl/+ samples in wet or dry conditions. 

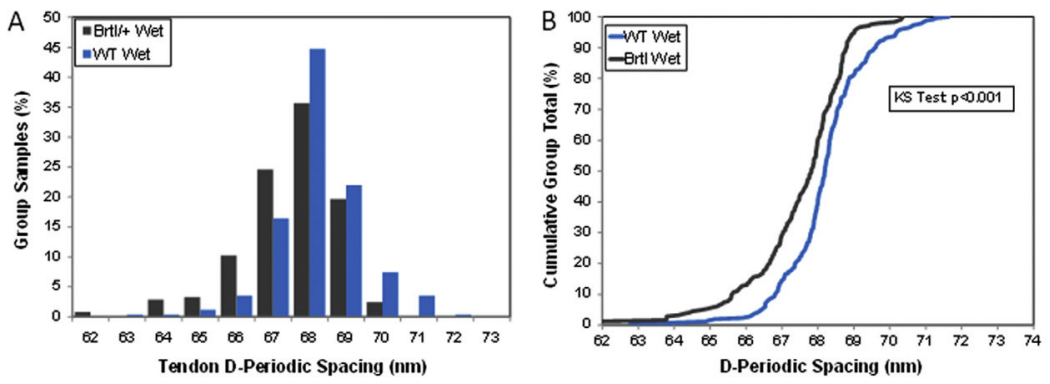

Fig. 4.

Histogram and cumulative distribution function for D-periodic spacing measured in wet samples. Panel A shows the histogram and panel B shows the Cumulative Distribution Function $(\mathrm{CDF})$ computed from the measurements in each group. Both the histogram and the CDF indicate that the Brtl/+ tendon populations were shifted downward in comparison to their wild type (WT) counterparts, although the shape of the 2 populations were similar. A Kolmogorov-Smirnov test, indicated a significant difference in populations in Brtl/+ versus WT tendons. 

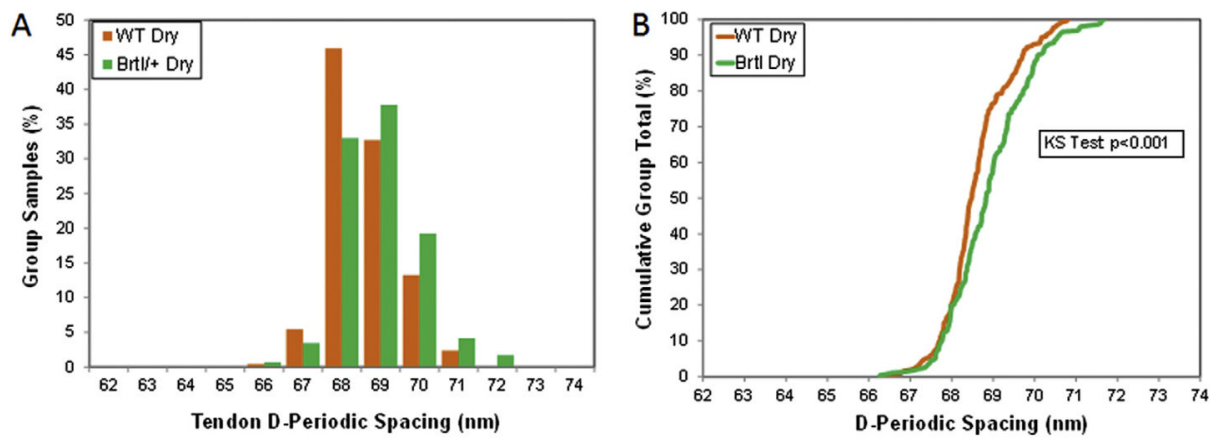

Fig. 5.

Histogram and cumulative distribution function for D-periodic spacing measured in dry samples. Panel A shows the histogram and panel B shows the Cumulative Distribution Function $(\mathrm{CDF})$ computed from the measurements in each group. A clear difference between histograms is not immediately apparent, although the Brtl/+ population may be slightly shifted upwards. The CDF indicates that the populations are very similar up to about $68 \mathrm{~nm}$, then the wild type (WT) population climbs while the Brtl/+ population is delayed, spreading values towards larger spacing. A $\mathrm{K}-\mathrm{S}$ test indicated that the $\mathrm{D}$-periodic spacings were significantly different between the two groups $(p<0.001)$. 

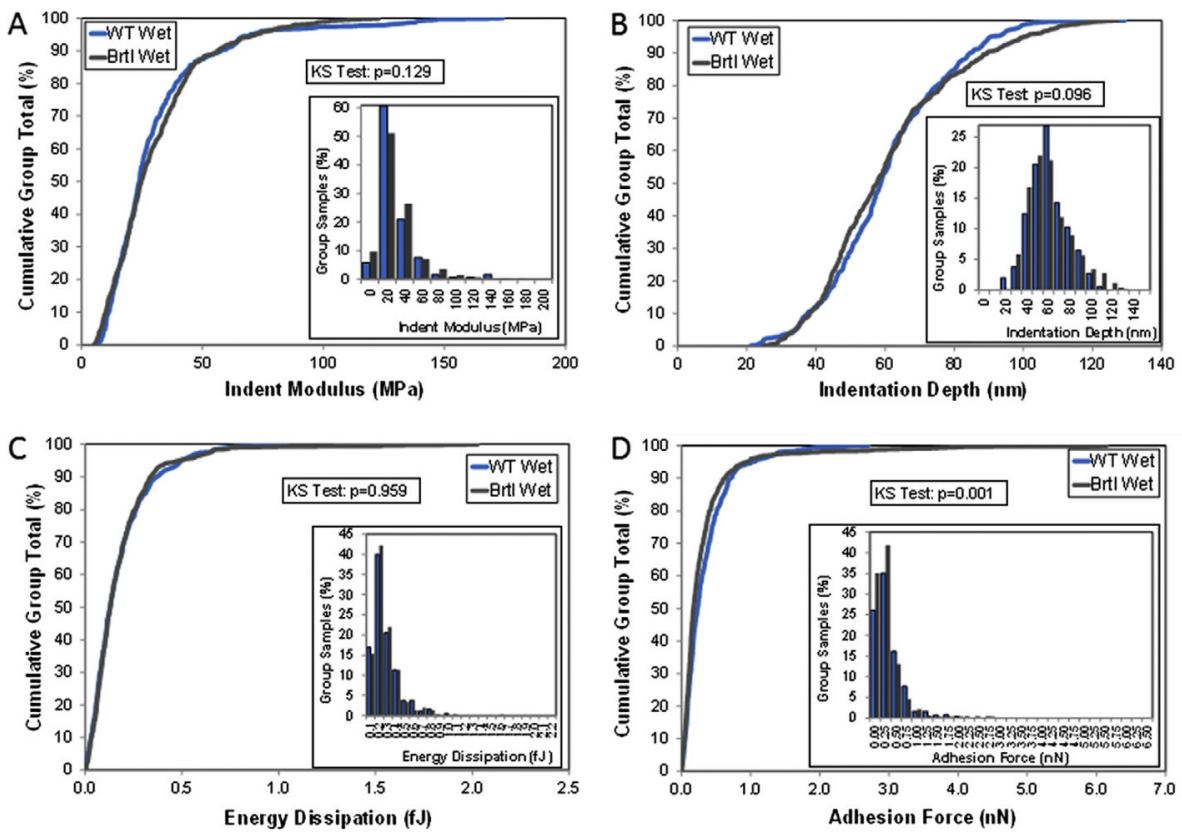

Fig. 6.

Mechanical measures from hydrated tendon samples. Mechanical comparisons were made for all indentations within each genotype ( $n=555$ for wild type (WT), $n=614$ for Brtl/ +). Histograms and cumulative distribution functions (CDFs) were produced for each property and statistically compared using KS tests There were no significant differences in elastic modulus (A), indentation depth (B) or energy dissipation (C) between WT and Brtl/+ samples. There was, however, a significant difference in adhesion force (D). 

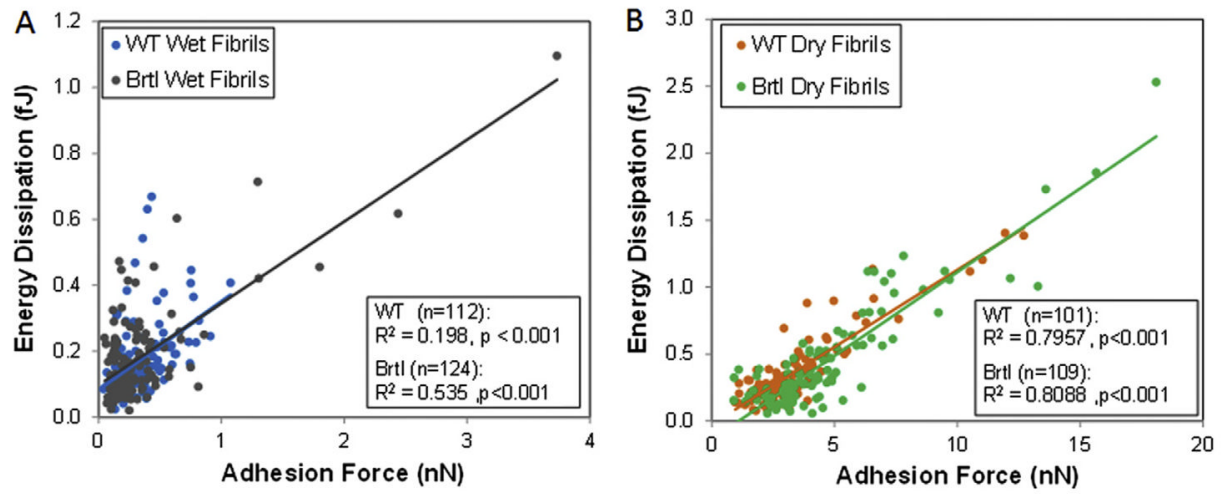

Fig. 7.

Linear regression between adhesion force (independent) and energy dissipation (dependent). In wild type (WT) and Brtl/+ fibrils, under hydrated and dry conditions, a strong and positive relationship existed between energy dissipation and adhesion force. This relationship suggests that adhesion may be the mechanism behind energy that is dissipated with loading. 

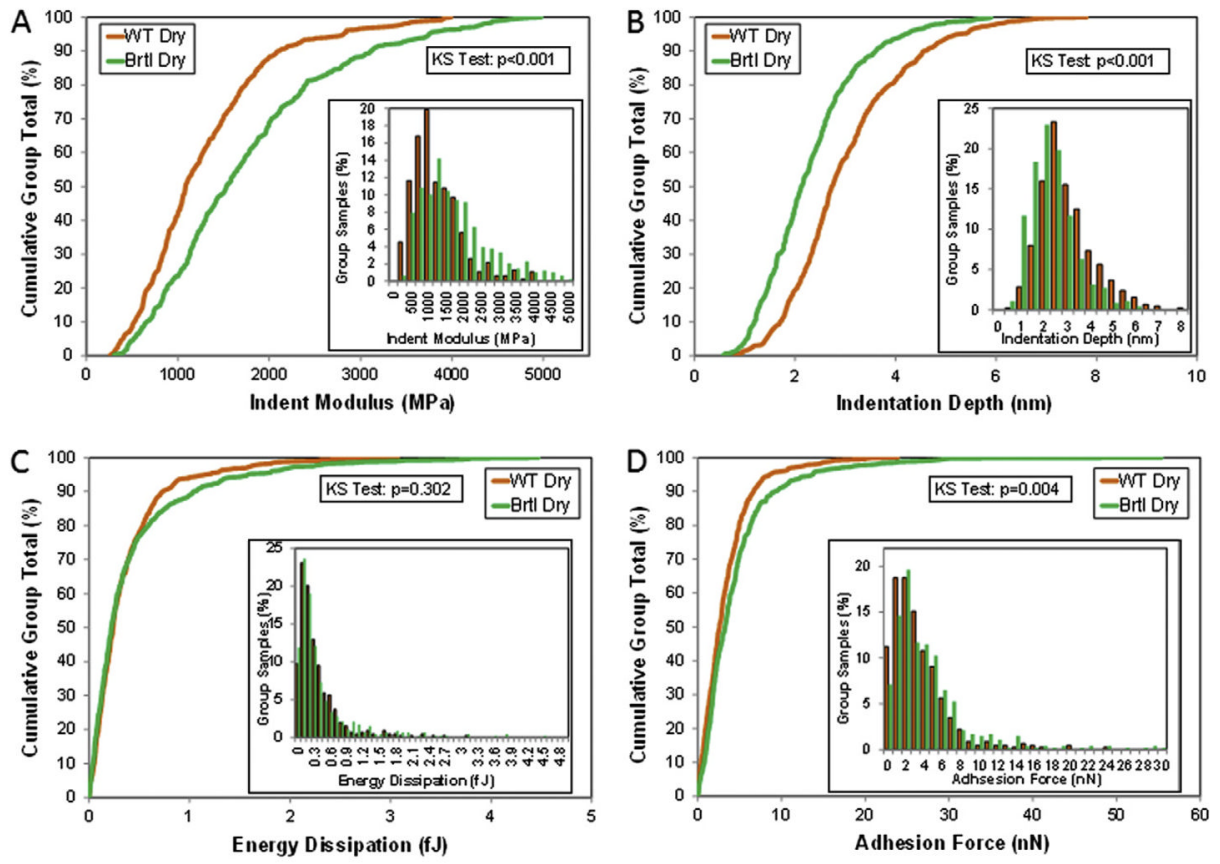

Fig. 8.

Mechanical measures from dehydrated tendon samples. Mechanical comparisons were made for all indentations within each genotype ( $n=464$ for wild type (WT), $n=479$ for Brtl/+). Histograms and cumulative distribution functions (CDFs) were produced for each property and statistically compared using $\mathrm{KS}$ tests As opposed to the wet condition, there were significant mean differences between the WT and Brtl/+ fibrils for elastic modulus (A), indentation depth (B) and adhesion force (D), but no difference for energy dissipation (D). Population differences were particularly compelling for modulus and indentation depth, where the Brtl/+ fibrils were on average $37 \%$ stiffer leading to $23 \%$ lower indentation depth for the same indentation force. 
Table 2

Correlations between D-spacing and mechanical measures from individual fibrils in wet samples $(n=112 \mathrm{WT}, n=124 \mathrm{Brtl} /+)$.

\begin{tabular}{llllll}
\hline & \multicolumn{2}{l}{ WT correlations (Wet) } & \multicolumn{2}{l}{ Brtl/+ correlations (Wet) } \\
\cline { 2 - 3 } & $\boldsymbol{r}$ value & $\boldsymbol{p}$ value & & $\boldsymbol{r}$ value & $\boldsymbol{p}$ value \\
\hline D-spacing vs. modulus & -0.027 & 0.776 & -0.081 & 0.369 \\
D-spacing vs. Indentation depth & -0.068 & 0.476 & 0.056 & 0.536 \\
D-spacing vs. energy dissipation & -0.237 & 0.012 & -0.047 & 0.608 \\
Modulus vs. energy dissipation & -0.289 & 0.002 & -0.294 & $<0.001$ \\
Indentation depth vs. energy dissipation & 0.409 & $<0.001$ & 0.342 & $<0.001$ \\
\hline
\end{tabular}




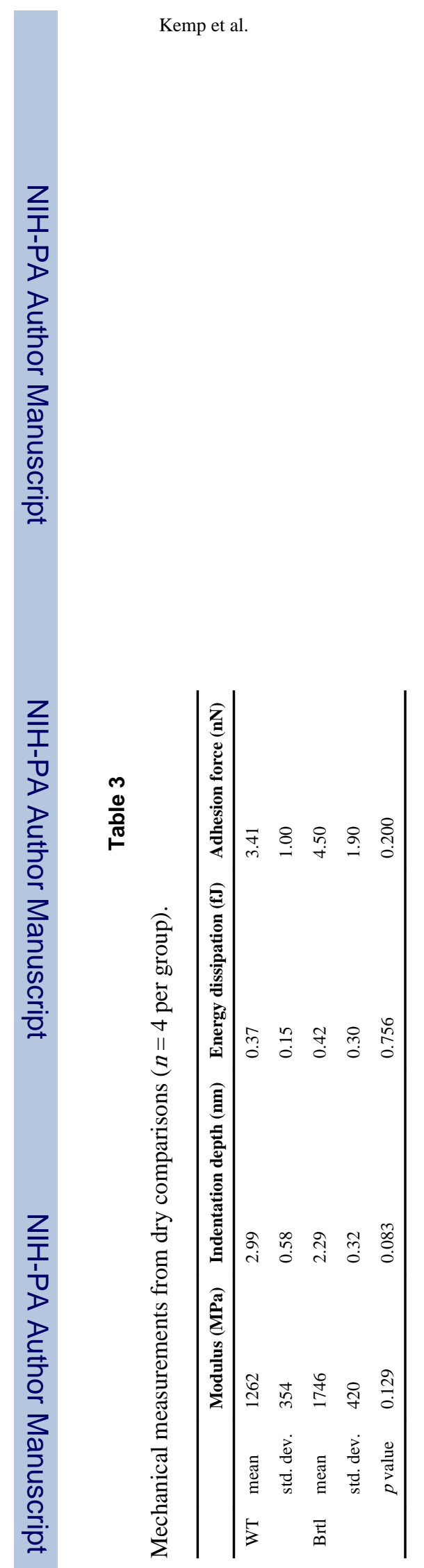

J Struct Biol. Author manuscript; available in PMC 2013 December 01. 
Table 4

Correlations between D-spacing and mechanical measures from individual fibrils in dry samples $(n=101 \mathrm{WT}, n=109 \mathrm{Brtl} /+)$.

\begin{tabular}{llllll}
\hline & \multicolumn{2}{l}{ WT correlations (Dry) } & \multicolumn{2}{l}{ Brtl/+ correlations (Dry) } \\
\cline { 2 - 3 } \cline { 5 - 6 } & $\boldsymbol{r}$ value & $\underline{\boldsymbol{p} \text { value }}$ & $\boldsymbol{r}$ value & $\boldsymbol{p}$ value \\
\hline D-spacing vs. modulus & 0.036 & 0.724 & 0.199 & 0.038 \\
D-spacing vs. indentation depth & -0.084 & 0.401 & -0.200 & 0.037 \\
D-spacing vs. energy dissipation & -0.140 & 0.162 & -0.174 & 0.070 \\
Modulus vs. energy dissipation & 0.095 & 0.343 & -0.330 & $<0.001$ \\
Indentation depth vs. energy dissipation & -0.130 & 0.195 & 0.330 & $<0.001$ \\
\hline
\end{tabular}


Table 5

Correlations between D-spacing and mechanical measures from all individual fibrils pooled $(n=446)$.

\begin{tabular}{llll}
\hline & $\boldsymbol{r}$ value & $\boldsymbol{r}^{\mathbf{2}}$ value & $\boldsymbol{p}$ value \\
\hline D-spacing vs. modulus & 0.298 & 0.089 & $<0.001$ \\
D-spacing vs. indentation depth & -0.266 & 0.071 & $<0.001$ \\
D-spacing vs. energy dissipation & 0.022 & 0.001 & 0.639 \\
Modulus vs. energy dissipation & 0.287 & 0.082 & $<0.001$ \\
Indentation depth vs. energy dissipation & -0.313 & 0.097 & $<0.001$ \\
Adhesion force vs. energy dissipation & 0.832 & 0.692 & $<0.001$ \\
\hline
\end{tabular}

